Dhaka Univ. J. Biol. Sci. 21(2): 125-130, 2012 (July)

\title{
MORPHOLOGY AND BIOLOGY OF THE BEDBUG, CIMEX HEMIPTERUS (HEMIPTERA: CIMICIDAE) IN THE LABORATORY
}

\author{
Humayun Reza Khan* and Md. Monsur Rahman \\ Department of Zoology, University of Dhaka, Dhaka-1000, Bangladesh \\ Key words: Bedbug, Cimex hemipterus, Biology, Morphology
}

\begin{abstract}
Adult bedbugs collected from Fazlul Huq Muslim Hall, University of Dhaka were identified as Cimex hemipterus Fabricius (Hemiptera: Cimicidae). The bedbug species was reared and its morphology and biology were studied in the laboratory at room temperature $28 \pm 4^{\circ} \mathrm{C}$ and $70 \pm 10 \% \mathrm{RH}$. The average incubation period of the eggs was $7.67 \pm 2.08$ days. Average nymphal duration was $53.67 \pm 2.52$ days. The five stadia (stage durations) of the five nymphs were $8.33 \pm 0.58,12.0 \pm 1.0,11.33 \pm 0.58,12.0 \pm 1.0$ and $10.0 \pm 1.0$ days, respectively. Average time required from egg laying to adult emergence was $61.67 \pm 3.21$ days.
\end{abstract}

\section{Introduction}

Bedbugs are blood feeding ecto-parasites of humans, chickens, bats, and occasionally domesticated animals ${ }^{(1)}$. There are two common species of bedbugs, Cimex lectularius Linnaeus and $C$. hemipterus Fabricius which have a wide distribution in tropical and subtropical countries(2). Cimex hemipterus known as 'Indian bedbug', is found in both rural and urban conditions in Bangladesh ${ }^{(3)}$. The bedbugs are gregarious and are frequently found in large numbers. They live under crowded and uncared for living conditions and often associated with army barracks, labour and prison camps and similar situations where they may readily contact a variety of hosts ${ }^{(4)}$. Within human dwellings, the bedbugs prefer cracks and crevices in walls, furniture, behind wall paper and wood paneling or under carpeting(5). During the day the bedbugs hide in cracks and crevices of furniture and debris, emerging at night to seek a blood meal (6). They are usually active during night, feed during the day when hungry ${ }^{(1)}$.

In the present paper the morphology and biology of the bedbug C. hemipterus was studied in the laboratory condition.

\section{Materials and Methods}

The infestation of the bedbug C. hemipterus is high in the student's residential halls of the University of Dhaka. The adult bedbugs were collected from the infested mattress of the students' beds of Fazlul Huq Muslim Hall of the University. The adult bedbugs were collected with the help of a soft brush and were kept into a beaker. They were reared in the Entomology Research Laboratory of the Department of Zoology, University of Dhaka

*Author for correspondence: <hrkha@hotmail.com>. 
at room temperature $\left(28 \pm 4^{\circ} \mathrm{C}\right)$ and $70 \pm 10 \% \mathrm{RH}$ to obtain a continuous supply of the bugs for the study. The experiment was conducted from May to August, 2007 in the laboratory.

The collected adult bedbugs (both males and females) were reared in glass beakers. A piece of cloth was kept inside the beaker for refuge and egg laying by the females. The opening end of the beaker was covered with a piece of fine cloth being tied with a piece of thread. The bugs were supplied with the blood meal of a pigeon. The breast feathers of the pigeon were removed and the glass beaker containing the bugs was held downward on the breast in order that the bugs could suck the pigeon blood. Both the male and female bugs were fed with pigeon blood at every alternate day.

The adult females started laying eggs on the pieces of the cloth kept inside the rearing beaker. The cloth with the eggs was then transferred to a new beaker daily and a new piece of cloth was placed inside the beaker. The time of the egg laying was recorded regularly.

The eggs on the cloth were hatched into the first instar nymphs, which were separated and taken into a new beaker with a soft brush. The incubation period between egg laying and egg hatching was recorded.

The newly hatched 1st instar nymphs were reared in a glass beaker containing a piece of cloth and were given pigeon blood meal as their food. Five successive moults occurred before adult emergence, i.e. the 5th instar nymphs emerged as adults. The moulting was determined by the caste off skin (exuvium) and size. In each moulting, the stadium was recorded as the numphal period in days between two successive moults.

\section{Results and Discussion}

Morphological description of adult bedbug: The body of the bed bug is oval shaped, flattened dorso-ventrally; when unfed, they were pale yellow or brown in colour, but after a full blood meal they became reddish brown. The average length and width of the adult bugs were 5.5 and $2.5 \mathrm{~mm}$, respectively (Table 1; Figs 1-2); length may reach up to eight millimeters.

The head was short, broad and pointed at the tip and had a pair of prominent compound eyes, in front of which present a pair of antennae. Compound eyes were oval, black and sessile. The antenna was four-segmented; the first segment was shorter than other segments; the 3rd and 4th segments were more slender and transparent than the first two segments. Fine hair like structures were present in all four segments of the antennae. Fine hairs were also found on the border of head except the position of compound eyes and antennae.

Mouth parts were piercing and sucking type located on the ventral side of the head. These consist of a triangular labrum, a long 3-segmented labium reaching at the base of prothorax and paired mandible and maxillary stylets, which were blade like. The mouth 
parts are modified for piercing the host skin and sucking the blood. The mandibular stylets make a puncture on the skin of the host and the maxillary stylets enter the wound. The saliva runs down into the puncture and prevents the clotting of blood which is subsequently sucked up.
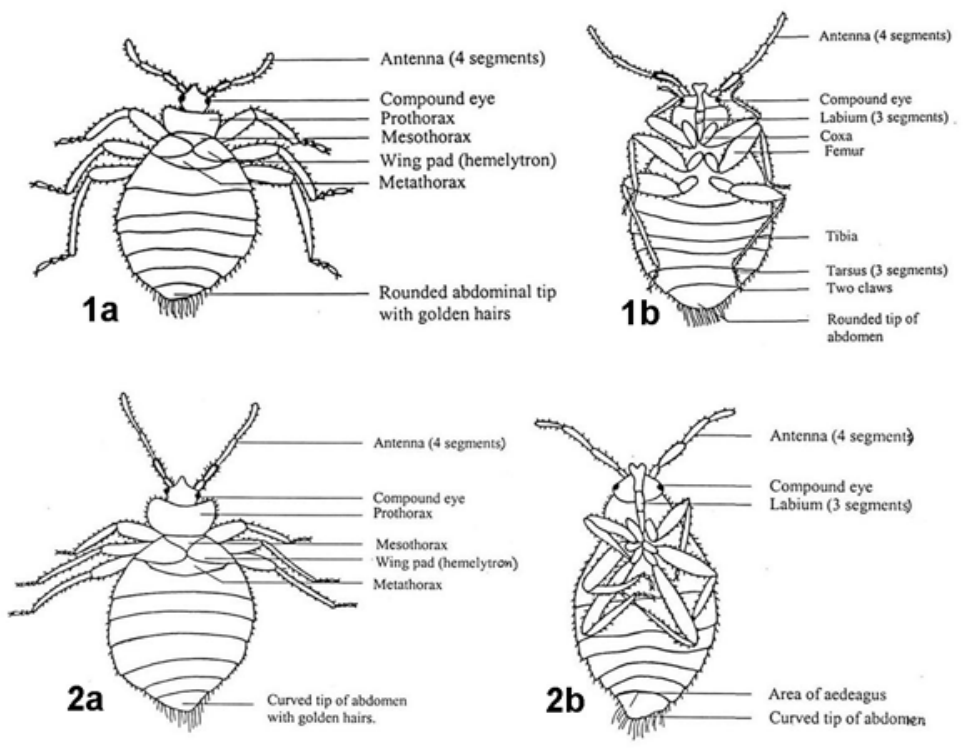

Figs 1-2: 1a. An adult female Cimex hemipterus (dorsal view). 1b. An adult female C. hemipterus (ventral view). 2a. An adult male C. hemipterus (dorsal view). 2b. An adult male C. hemipterus (ventral view).

Table 1. Average time required to complete different immature stages of $C$. hemipterus at room temperature $\left(28 \pm 4^{\circ} \mathrm{C}\right)$ and $70 \pm 10 \% \mathrm{RH}$.

\begin{tabular}{lcccccccc}
\hline $\begin{array}{l}\text { No. of } \\
\text { observa- } \\
\text { tion }\end{array}$ & $\begin{array}{c}\text { Incuba } \\
\text {-tion } \\
\text { period } \\
\text { (days) }\end{array}$ & $\begin{array}{c}\text { 1st } \\
\text { instar }\end{array}$ & $\begin{array}{c}\text { 2nd } \\
\text { instar }\end{array}$ & $\begin{array}{c}\text { 3rd } \\
\text { instar }\end{array}$ & $\begin{array}{c}\text { 4th } \\
\text { instar }\end{array}$ & $\begin{array}{c}5 \text { th } \\
\text { instar }\end{array}$ & $\begin{array}{c}\text { Total stage } \\
\text { duration of } \\
\text { nymphs } \\
\text { (days) }\end{array}$ & $\begin{array}{c}\text { Life cycle } \\
\text { duration from } \\
\text { egg to adult } \\
\text { (days) }\end{array}$ \\
\hline 1 & 6 & 6 & 11 & 10 & 11 & 9 & 47 & 53 \\
2 & 10 & 7 & 13 & 10 & 12 & 8 & 50 & 60 \\
3 & 7 & 9 & 10 & 13 & 14 & 11 & 57 & 64 \\
Mean \pm Sd & $7.67 \pm$ & $7.33 \pm$ & $11.33 \pm$ & $7.67 \pm$ & $12.33 \pm$ & $9.33 \pm$ & $51.33 \pm$ & $59.00 \pm$ \\
& 2.88 & 1.53 & 1.53 & 1.73 & 1.53 & 1.53 & 5.13 & 5.57 \\
\hline
\end{tabular}

The thorax is three segmented. The prothorax was much larger than the mesothorax and the metathorax, and had a distinct wing like expansion. The prothorax was found generally twice as wide as its length. Fine hairs were found on he boarder of the prothorax. The mesothorax was a triangular fold and the metathorax, crescentric. Wings are absent. Each thoracic segment contained a pair of jointed walking legs, there were thus three pairs of legs. Each leg consisted of a linear series of segments, namely coxa, 
trochanter, femur, tibia and tarsus. The coxa was stout, flattened and short by which the leg was articulated with the thorax. The trochanter was small triangular structure fused to femur, which was broader, tubular and the strongest part of the leg. The tibia was slender and longer than other segments. The tarsus was 3-segmented of which two were more or less equal in size and the third one was longer. Two claws were at the tip of tarsus. Femur and tibia were covered with fine spines. The legs of the bedbugs are not adapted for clinging, but rather allow for rapid movement over the host's body. On the ventral side of the third thoracic somite is a pair of scent glands. These glands produce an oily secretion that is thought to be a defense mechanism against predators.

The abdomen was eight segmented. The 1st and 2nd abdominal segments were fused together. In adult male bed bug, the abdomen was narrower and its tip was curved and slightly more pointed than in the female. A small curved aedeagus was seen ventrally at the tip of the male abdomen. In the adult female, the abdomen was broad and rounded. A small incision was seen on the left side of the 4th abdominal segment of the female. This incision is the opening of a blind copulatory pouch known as the organ of Berlese. The entire abdomen was covered with numerous small hairs on both dorsal and ventral sides. At the tip of the abdomen of both the male and female bed bugs, there presents a tuft of relatively longer and golden hairs. Females are negligibly longer and wider than males, although their sensory bristles are some what shorter

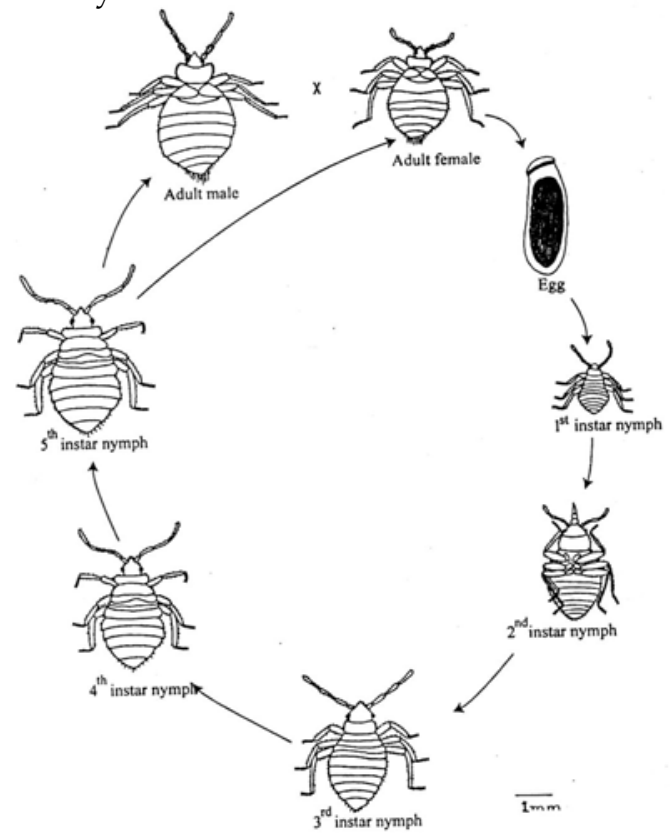

Fig. 3. Life cycle of Cimex hejmipterus.

The life cycle of the bed bug is shown in Fig. 3. The life cycle of the bedbugs consists of egg, five instars of nymphs and adults. The description of these stages is given below. 
In the laboratory, the eggs (Figs $4 a, b$ ) were laid on the cloths placed inside the rearing beaker. They were glued to the cloths. They were creamy white, cylindrical and slightly curved interiorly. The colour of the eggs changed to yellowish-white before hatching into nymph. The average length of each egg was one millimeter which was also observed by Service(2). One end of the eggs was round and blind, and the other end was curved and provided with a cap-like operculum (Fig. 2). Inside the eggs an impression of elongated-oval embryo was seen from the outside.

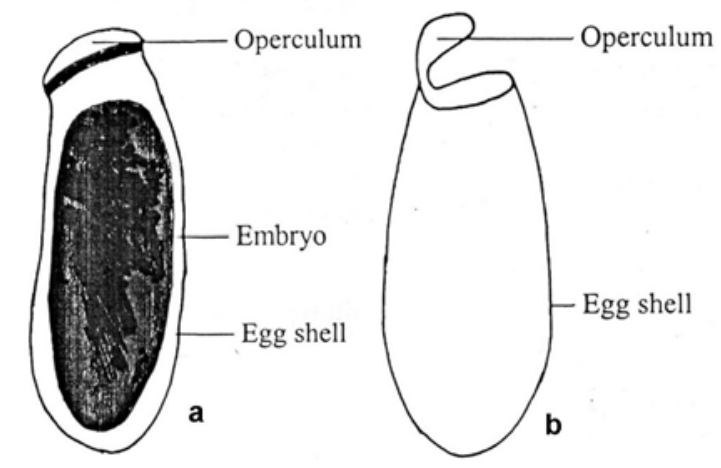

Fig. 4a-b. a. An unhatched egg of $C$. hemipterus.

b. An egg shell of C. hemipterus after hatching.

The average incubation period of the eggs of the laboratory reared $C$. hemipterus was $7.67 \pm 2.08$ days (Table 1 ). The eggs usually hatch after about $8-11$ days, but the hatching takes place within less than a week if temperature is about $27^{\circ} \mathrm{C}^{(2)}$ which is almost in conformity with the present findings (Av. 7.67 days) in the laboratory condition at an average temperature of $28^{\circ} \mathrm{C}$. Service( ${ }^{(2)}$ also reported that at low temperature the eggs of C. hemipterus can survive for up to three months, but hatching does not occur below $13^{\circ} \mathrm{C}$.

The 1st instar nymph (Fig. 5) was pale yellow in colour when unfed and average length was $1.5 \mathrm{~mm}$. After feeding a blood meal, the colour of the body turned into red resembling a blood droplet. The $2^{\text {nd }}$ instar nymph (Fig. 6) was pale yellow in colour when unfed, after feeding a blood meal, the body colour was red, and average length was 2.0 $\mathrm{mm}$. The 3rd instar nymph (Fig. 7) was slightly darker than the 2nd instar nymph, colour of the body after blood-fed was red; and the average length of the nymph was $2.5 \mathrm{~mm}$. The 4th instar nymph (Fig. 8) was brown in unfed condition and red after blood feeding; average length was $3.0 \mathrm{~mm}$. Mesothorax and metathorax were slightly curved. The 5th instar nymph (Fig. 9) was blackish-brown in colour in unfed condition and red in bloodfed condition; average length was $4.5 \mathrm{~mm}$. Wings pads or hemelytra started to develop on the mesothorax. After moulting, it emerged into an adult bedbug. 

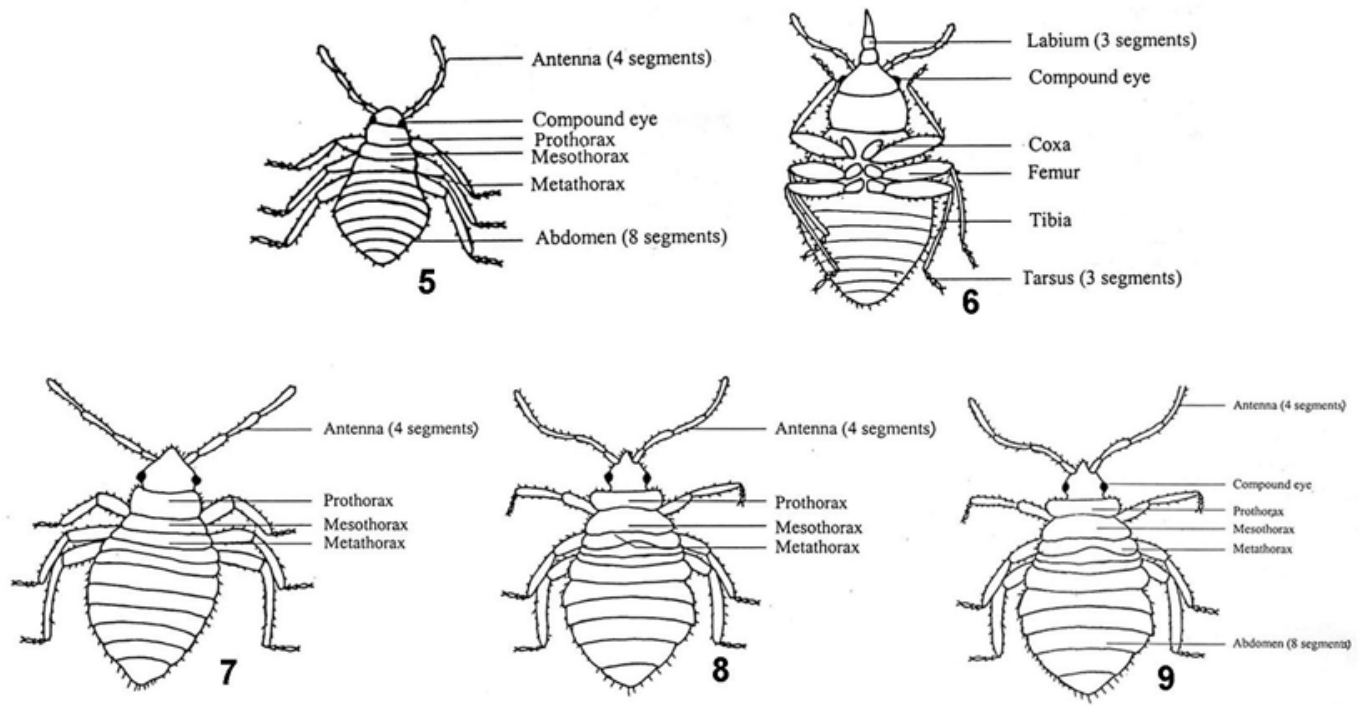

Figs 5-9. 5. 1st instar nymph of $C$. hemipterus (dorsal view). 6. 2nd instar nymph of C. hemipterus (ventral view). 7. 3rd instar nymph of C. hemipterus (dorsal view). 8. 4th instar nymph of $C$. hemipterus (ventral view). 9. 5th instar nymph of $C$. hemipterus (dorsal view).

The average total nymphal period from the 1st to the 5 th instar nymphs was $51.33 \pm$ 5.13 days; the stadia of the five nymphal instars were $7.33 \pm 1.53,11.33 \pm 1.53,7.67 \pm 1.73$, and $12.33 \pm 1.52$ and $9.33 \pm 1.53$ days, respectively. Average time required from egg laying to adult emergence was $59 \pm 5.57$ days. (Table 1 ). The nymphal period commonly last 5 - 8 weeks (35 - 56 days), but may be greatly extended in cool conditions or if regular blood- feeding is prevented by lack of hosts ${ }^{(2)}$.

\section{References}

1. Usinger RL 1966. Monograph of Cimicidae (Hemiptera- Heteroptera). Entomological Society of America, College Park, Maryland, USA.

2. Service MW 1996. Medical Entomology for students. Chapman and Hall, London, pp. 202-206.

3. Ahmed TU and M Begum 1992. Bangladesher Janoshaster Anisthokari pokamakor (in Bangla). Bangla Academy, Dhaka, Bangladesh, pp. 99-102.

4. Metcalf CL and WP Flint 1973. Destructive and useful insects: their habits and control (4 $4^{\text {th }}$ edition). Tata McGraw Hill Publishing Company Ltd., New Delhi, pp. 1011-1012.

5. Krueger L 2000. Don't get bitten by the resurgence of bed bugs. Pest Control 68: 58-64.

6. Ross HH 1965. A Text Book of Entomology. Toppan Co. Ltd. Tokyo, Japan, pp. 287. 